\title{
Effect of Azilsartan on Day-to-Day Variability in Home Blood Pressure: A Prospective Multicenter Clinical Trial
}

\author{
Toru Miyoshi ${ }^{\mathrm{a}, \mathrm{f}}$, Ryoji Suetsuna ${ }^{\mathrm{b}}$, Naoto Tokunaga ${ }^{\mathrm{c}}$, Masayasu Kusaka ${ }^{\mathrm{b}}$, Ryuichiro Tsuzaki ${ }^{\mathrm{d}}$, \\ Kazuya Koten ${ }^{\mathrm{e}}$, Kohno Kunihisa ${ }^{\mathrm{a}}$, Hiroshi Ito ${ }^{\mathrm{a}}$
}

\begin{abstract}
Background: The blood pressure variability (BPV) such as visit-tovisit, day-by-day, and ambulatory BPV has been also shown to be a risk of future cardiovascular events. However, the effects of antihypertensive therapy on BPV remain unclear. The purpose of this study was to evaluate the effect of azilsartan after switching from another angiotensin II receptor blocker (ARB) on day-to-day BPV in home BP monitoring.
\end{abstract}

Methods: This prospective, multicenter, open-labeled, single-arm study included 28 patients undergoing treatment with an ARB, which was switched to azilsartan after enrollment. The primary outcome was the change in the mean of the standard deviation and the coefficient of variation of morning home BP for 5 consecutive days from baseline to the 24-week follow-up. The secondary outcome was the change in arterial stiffness measured by the cardio-ankle vascular index.

Results: The mean BPs in the morning and evening for 5 days did not statistically differ between baseline and 24 weeks. For the morning $\mathrm{BP}$, the means of the standard deviations and coefficient of variation of the systolic BP were significantly decreased from $7.4 \pm 3.6 \mathrm{~mm} \mathrm{Hg}$ to $6.1 \pm 3.2 \mathrm{~mm} \mathrm{Hg}$ and from $5.4 \pm 2.7 \%$ to $4.6 \pm 2.3 \%$ (mean \pm standard deviation, $\mathrm{P}=0.04$ and $\mathrm{P}=0.04$, respectively). For the evening $\mathrm{BP}$, no significant change was observed in the systolic or diastolic BPV. The cardio-ankle vascular index significantly decreased from $8.3 \pm 0.8$ to $8.1 \pm 0.8(\mathrm{P}=0.03)$.

Conclusions: Switching from another ARB to azilsartan reduced day-to-day BPV in the morning and improved arterial stiffness.

Keywords: Day to day; Variability; Azilsartan; Clinical trial; Arterial

Manuscript accepted for publication April 27, 2017

aDepartment of Cardiovascular Medicine, Okayama University Graduate School of Medicine, Density and Pharmaceutical Sciences, Okayama, Japan bepartment of Internal Medicine, Kusaka Hospital, Okayama, Japan 'Department of Cardiovascular Medicine, Kaneda Hospital, Okayama, Japan dDepartment of Internal Medicine, Niimi Central Hospital, Okayama, Japan 'Sugie Clinic, Okayama, Japan

${ }^{f}$ Corresponding Author: Toru Miyoshi, Department of Cardiovascular Medicine, Okayama University, 2-5-1, Shikata-cho, Kita-ku, Okayama 700-8558, Japan. Email: miyoshit@cc.okayama-u.ac.jp

doi: https://doi.org/10.14740/jocmr3050w stiffness

\section{Introduction}

Studies have consistently demonstrated that a higher mean blood pressure (BP) is associated with a higher risk of allcause mortality and cardiovascular morbidity and mortality [1, 2]. However, BP does not remain steady but instead fluctuates continually within a $24-\mathrm{h}$ period; this occurs from day to day and from month to month $[3,4]$. This BP variability (BPV) has also been shown to be a risk factor for future cardiovascular events and mortality [5-7]. Therefore, it is of great clinical interest to determine whether BPV can serve as a target of BP-lowering therapy and to identify effective antihypertensive medications that can effectively reduce BPV. However, several studies have shown that various antihypertensive drug classes differentially influence BPV $[8,9]$.

A large amount of evidence indicates that angiotensin II receptor blockers (ARBs) reduce cardiovascular events in patients with hypertension [10]. Azilsartan, which is a recently approved ARB, reportedly has greater antihypertensive effects than other ARBs [11, 12]. In addition, several studies have shown that azilsartan has pleiotropic effects such as improvement in cardiometabolic parameters [13-16]. Thus, azilsartan is a promising $\mathrm{ARB}$ for primary and secondary prevention of cardiovascular disease.

The purpose of this prospective study was to evaluate the effect of azilsartan after switching from another ARB on dayto-day BPV derived from home BP monitoring.

\section{Materials and Methods}

\section{Patient population}

Participants were enrolled at six affiliated hospitals or clinics of Okayama University in Japan from July 2013 to June 2015. The study was approved by the ethics committees of all hospitals and clinics. All participants provided written informed consent before enrollment. This study was conducted according to the principles expressed in the Declaration of Helsinki. The study is registered in the UMIN Clinical Trials Registry 


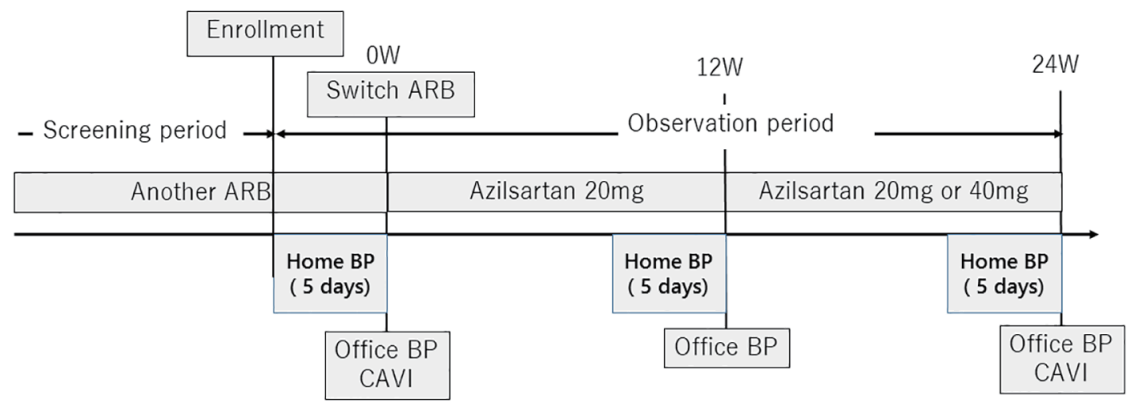

Figure 1. Study protocol. ARB: angiotensin II receptor blocker; BP: blood pressure; BPV: blood pressure variability.

\section{(UMIN000012586).}

Eligible patients were adults $(>30$ and $<85$ years old $)$ who were undergoing treatment with an ARB (excluding azilsartan) but did not achieve the target BP according to the Japanese Society of Hypertension Guidelines for the Management of Hypertension 2009 (JSH2009) [17]. Patients with the following conditions were excluded: secondary hypertension, grade III hypertension according to the JSH 2009 guideline [17], congestive heart failure, severe valvular heart disease, myocardial infarction or cerebellar infarction within 6 months, chronic atrial filtration, malignant arrhythmia, renal insufficiency (serum creatinine concentration $\geq 2 \mathrm{mg} / \mathrm{dL}$ ), severe liver disease, chronic inflammatory disease, malignant disease (lifetime prognosis $<6$ months), and treatment with a reninangiotensin system inhibitor other than an ARB.

\section{Study protocol}

The AziLsartan treatMent as a swItch strateGy for improvement of uncontrolled Hypertension and blood pressure variabiliTY (ALMIGHTY) was a 24-week, prospective, multicenter, open-label study with a single treatment arm (Fig. 1). Eligible subjects were assigned to receive azilsartan at $20 \mathrm{mg}$, and after 12 weeks, dose titration was permitted up to $40 \mathrm{mg}$ if BP lowering was insufficient according to the JSH 2009 guideline [17]. Treatment was continued for 24 weeks. Other drugs that could potentially interfere with the efficacy of the study medications were not allowed. All patients were reviewed for general health and compliance with medication, which was assessed by tablet counts and BP checks at each office visit.

The primary outcome was the change in the day-to-day home morning BPV. The day-to-day home BPV was defined as the standard deviation (SD) and the coefficient of variation (CV) of the SD of BP on 5 consecutive days (SD and CV of 5 day-to-day readings) $[18,19]$. The $\mathrm{CV}$ was defined as the within-individual SD divided by the mean BP. To avoid a multiplicity issue, we prioritized the SD. The secondary outcome was the change in arterial stiffness, which was measured by the cardio-ankle vascular index (CAVI).

Our calculations revealed that a sample of 210 patients would provide a power of $95 \%$ and type I error of $5 \%$ to detect $\mathrm{a} \geq 1-\mathrm{mm} \mathrm{Hg}$ decrease in the primary outcome. We assumed that the SD of the day-to-day home BPV would decrease from $8 \mathrm{~mm} \mathrm{Hg}$ (SD, $4 \mathrm{~mm} \mathrm{Hg}$ ) to $7 \mathrm{~mm} \mathrm{Hg}$ (SD, $4 \mathrm{~mm} \mathrm{Hg}$ ) [20].

\section{Measurement of clinic and home BP}

Office BP was measured in a sitting position three times at 1-min intervals using a validated oscillometric device after an initial 5 min of seated rest. Home BP was measured in a sitting position three times each morning and three times each evening for 5 consecutive days before the office visit (total of three visits) using a validated oscillometric device. The subjects were instructed to rest for $5 \mathrm{~min}$ before the first reading and rest for a 15-s interval between readings. Morning BP was measured within $1 \mathrm{~h}$ after waking, after urination, and before breakfast. Evening BP was measured just before going to bed and $\geq 60$ min after taking a bath according to Japanese home BP guidelines [21].

\section{Arterial stiffness measurement}

To assess arterial stiffness, the CAVI was measured automatically using a VaSera (Fukuda Denshi, Tokyo, Japan) from the BP and pulse wave velocity (PWV) measurements while monitoring the electrocardiogram and heart sounds [22]. The PWV was calculated by dividing the distance from the aortic valve to the ankle artery by the sum of the time between the aortic valve closing sound and the notch of the brachial pulse wave and the time between the rise of the brachial pulse wave and the ankle pulse wave. The CAVI was determined using the following equation: $\mathrm{CAVI}=\mathrm{a}((2 \rho / \Delta \mathrm{P}) \times \ln (\mathrm{Ps} / \mathrm{Pd})$ $\left.\times \mathrm{PWV}^{2}\right)+\mathrm{b}$, where Ps and $\mathrm{Pd}$ are the systolic and diastolic $\mathrm{BP}$, respectively; PWV is the PWV between the heart and ankle; $\Delta \mathrm{P}$ is Ps - Pd; $\rho$ is the blood density; and a and $\mathrm{b}$ are constants. The average of right and left CAVI was used for analysis.

\section{Statistical analysis}

Continuous variables are presented as mean $\pm \mathrm{SD}$, and categorical variables are presented as absolute value and proportion (\%). Variables at 3 and 6 months were compared with baseline data using a paired $t$-test. The threshold level for statistical significance was established at $\mathrm{P}<0.05$ (two-sided test). Statistical analysis was performed using SPSS 24.0 for Windows (IBM Corp., Armonk, NY, USA). 


\section{Results}

\section{Characteristics of the study participants}

During the entry period, 36 patients were enrolled in this study. Of them, 28 patients completed the study (eight patients were lost to follow-up). Table 1 shows the baseline characteristics of all subjects. Their mean age was 69 years, and $46 \%$ were men. The proportion of patients with diabetes mellitus and dyskinesia were $17 \%$ and $43 \%$, respectively. The ARBs taken by the participants before starting this study were candesartan $4 \mathrm{mg}$ (67\%), losartan $50 \mathrm{mg}$ (4\%), olmesartan $20 \mathrm{mg}$ (21\%), valsartan $80 \mathrm{mg}(4 \%)$, and telmisartan $40 \mathrm{mg}$ (4\%). All patients received an $\mathrm{ARB}$ with or without one other drug.

\section{Effects of azilsartan on day-to-day home BPV}

The office and home BPs did not change significantly between baseline and follow-up (Table 2). Table 3 shows the day-today home BPV. For the morning BP, the mean of the SD of the systolic BPV was significantly lower at 12 and 24 weeks than at baseline ( $\mathrm{P}=0.04$ and $\mathrm{P}=0.04$, respectively). The mean of the CV of the systolic BPV was also significantly at 12 and 24 weeks lower than baseline $(\mathrm{P}=0.04$ and $\mathrm{P}=0.04)$. The mean of the SD of the diastolic BPV was significantly lower at 12 and 24 weeks than at baseline $(\mathrm{P}=0.04$ and $\mathrm{P}=0.03$, respectively). The mean of the $\mathrm{CV}$ of the diastolic BPV was significantly lower than baseline only at 12 weeks $(P=0.04)$. For the evening BP, however, no significant changes were observed in the systolic and diastolic BPVs.

\section{Change in arterial stiffness}

Of the patients who completed this study, 18 patients underwent CAVI measurement at baseline and at follow-up. The
Table 1. Baseline Characteristics

\begin{tabular}{|c|c|}
\hline Age, years & $69 \pm 11$ \\
\hline Men, n (\%) & $13(46 \%)$ \\
\hline Body mass index $\left(\mathrm{kg} / \mathrm{m}^{2}\right)$ & $24.2 \pm 3.6$ \\
\hline Diabetes mellitus, n (\%) & $3(17 \%)$ \\
\hline Dyslipidemia, n (\%) & $12(43 \%)$ \\
\hline Chronic kidney disease, n (\%) & $5(18 \%)$ \\
\hline History of coronary artery disease, $\mathrm{n}(\%)$ & $2(7 \%)$ \\
\hline History of cerebrovascular disease, $\mathrm{n}(\%)$ & $2(7 \%)$ \\
\hline Current smoker, n (\%) & $1(4 \%)$ \\
\hline \multicolumn{2}{|c|}{ Angiotensin receptor blockers before enrollment } \\
\hline Candesartan 4 mg, n (\%) & $19(67 \%)$ \\
\hline Losartan 50 mg, n (\%) & $1(4 \%)$ \\
\hline Olmesartan 20 mg, n (\%) & $6(21 \%)$ \\
\hline Valsartan 80 mg, n (\%) & $1(4 \%)$ \\
\hline Telmisartan 40 mg, n (\%) & $1(4 \%)$ \\
\hline \multicolumn{2}{|l|}{ Concomitant medications } \\
\hline Calcium channel blockers, n (\%) & $11(39 \%)$ \\
\hline Diuretics, n (\%) & $2(7 \%)$ \\
\hline Beta blockers, n (\%) & $5(18 \%)$ \\
\hline
\end{tabular}

Data are expressed as mean \pm standard deviation or number (\%).

CAVI was significantly decreased from $8.3 \pm 0.8$ to $8.1 \pm 0.8$ $(\mathrm{P}=0.03)$.

\section{Discussion}

To the best of our knowledge, this is the first study to evaluate the effect of azilsartan after switching from another ARB on the day-to-day variability in home BP. Azilsartan treatment

Table 2. Mean Office and Home Blood Pressures

\begin{tabular}{|clll}
\hline & Baseline & 12 weeks & 24 weeks \\
\hline Office & & & \\
\hline Systolic BP (mm Hg) & $140.3 \pm 15.7$ & $139.3 \pm 19.4$ & $136.8 \pm 16.0$ \\
Diastolic BP (mm Hg) & $80.3 \pm 13.7$ & $79.8 \pm 12.3$ & $76.8 \pm 11.2$ \\
\hline Heart rate (beats/min) & $77.3 \pm 12.6$ & $76.1 \pm 12.2$ & $74.6 \pm 10.7$ \\
\hline Home (morning) & & & \\
\hline Systolic BP (mm Hg) & $136.9 \pm 13.1$ & $136.2 \pm 12.0$ & $133.3 \pm 10.0$ \\
Diastolic BP (mm Hg) & $79.9 \pm 10.2$ & $79.2 \pm 9.9$ & $79.6 \pm 9.6$ \\
HR (beats/min) & $67.4 \pm 7.6$ & $68.2 \pm 8.6$ & $67.1 \pm 7.1$ \\
\hline Home (evening) & & & \\
\hline Systolic BP (mm Hg) & $134.2 \pm 16.6$ & $130.1 \pm 14.3$ & $130.2 \pm 10.8$ \\
Diastolic BP (mm Hg) & $76.3 \pm 11.8$ & $75.4 \pm 10.3$ & $73.2 \pm 9.0$ \\
\hline Heat rate (beats/min) & $67.5 \pm 6.2$ & $69.5 \pm 6.2$ & $64.2 \pm 6.7$ \\
\hline
\end{tabular}

Data are expressed as mean \pm standard deviation. BP: blood pressure. 
Table 3. Day-to-Day Home Blood Pressure Variability

\begin{tabular}{|c|c|c|c|}
\hline & Baseline & 12 weeks & 24 weeks \\
\hline \multicolumn{4}{|l|}{ Morning } \\
\hline \multicolumn{4}{|l|}{ Systolic BP } \\
\hline $\mathrm{SD}(\mathrm{mm} \mathrm{Hg})$ & $7.4 \pm 3.6$ & $6.3 \pm 3.3^{*}$ & $6.1 \pm 3.2 *$ \\
\hline $\mathrm{CV}(\%)$ & $5.4 \pm 2.7$ & $4.6 \pm 2.4^{*}$ & $4.6 \pm 2.3^{*}$ \\
\hline \multicolumn{4}{|l|}{ Diastolic BP } \\
\hline $\mathrm{SD}(\mathrm{mm} \mathrm{Hg})$ & $4.8 \pm 2.8$ & $3.9 \pm 1.7^{*}$ & $3.6 \pm 1.9 *$ \\
\hline $\mathrm{CV}(\%)$ & $5.9 \pm 3.5$ & $4.8 \pm 2.3^{*}$ & $4.5 \pm 2.4$ \\
\hline \multicolumn{4}{|l|}{ Evening } \\
\hline \multicolumn{4}{|l|}{ Systolic BP } \\
\hline $\mathrm{SD}(\mathrm{mm} \mathrm{Hg})$ & $6.9 \pm 3.7$ & $6.2 \pm 3.1$ & $6.2 \pm 3.2$ \\
\hline CV (\%) & $5.1 \pm 2.5$ & $4.8 \pm 2.2$ & $4.8 \pm 2.4$ \\
\hline \multicolumn{4}{|l|}{ Diastolic BP } \\
\hline $\mathrm{SD}(\mathrm{mm} \mathrm{Hg})$ & $4.2 \pm 2.4$ & $4.0 \pm 2.0$ & $4.1 \pm 2.2$ \\
\hline CV $(\%)$ & $5.5 \pm 2.6$ & $5.3 \pm 2.6$ & $5.6 \pm 3.0$ \\
\hline
\end{tabular}

Data are expressed as mean \pm SD. BP: blood pressure; SD: standard deviation; CV: coefficient of variation. ${ }^{*} \mathrm{P}<0.05$ vs. baseline.

for 24 weeks significantly reduced the day-to-day BPV in the morning. Azilsartan also decreased the arterial stiffness as determined by the CAVI, suggesting the mechanism underlying the reduction in home BPV.

Several previous studies have shown the effect of antihypertensive therapy on day-to-day BPV. However, the results were controversial. Ishikura et al [23] reported that use of an ARB without taking a calcium channel blocker increased the day-to-day BPV. However, Matsui et al [20] reported that the combined use of an ARB with a calcium channel blocker reduced the day-to-day BPV. Another group showed that alphaadrenergic inhibitors also reduce day-to-day BPV [24]. A recent large prospective study of home BP showed that the effects of antihypertensive drug therapy on BPV did not differ among calcium channel blockers, angiotensin converting enzyme inhibitors, and ARBs [25]. However, azilsartan is a recently approved ARB. Experimental studies have demonstrated that azilsartan has an antioxidative effect [13], improves the metabolic profile $[14,15]$, and restores endothelial function [16]. In addition, Ishiguro et al [26] reported the possibility that azilsartan contributes to regression of arteriosclerosis in rats. In fact, our data showed a decrease in arterial stiffness by azilsartan. Thus, improvements in metabolic factors may reduce activation of the sympathetic nervous system and lead to amelioration of baroreflex sensitivity. Taken together, these findings indicate that the decrease in peripheral resistance induced by azilsartan may contribute to a reduction in the day-to-day BPV.

The Ohasama study [6] and the Finn-Home study [27] showed that an increased day-to-day BPV is a predictor of cardiovascular events independent of the mean BP. Several cross-sectional studies have shown that day-to-day BPV is associated with target organ damage such as left ventricular hypertrophy [28] and proteinuria [24]. One study demonstrated that day-to-day BPV was more closely associated with im- provement in left ventricular hypertrophy and arterial stiffness as represented by the PWV than were ambulatory BPV and visit-to-visit BPV [29]. However, a recent large prospective interventional study demonstrated that day-to-day BPV had no impact on prognosis in patients with mild to moderate hypertension [25]. Further studies to evaluate the clinical benefit of day-to-day BVP for risk assessment of future cardiovascular events are warranted.

\section{Limitations}

This study has several limitations. First, it was an open-labeled, single-arm trial and may thus have potentially biased results. Second, the planned sample size was not achieved, and the study was underpowered. However, we found a significant change in the primary outcome even in this sample number, which may mitigate the influence of the lower-powered statistical test on the conclusion of the study. Third, the 5-day home BP measurement period may have been too short to accurately estimate the day-to-day home BPV, and this may have affected the results. Fourth, this study evaluated the SD and CV as parameters of the day-to-day home BPV. However, recent studies suggest the use of other parameters such as the average real variability [30] or variability independent of the mean [3] to avoid the influence of the mean BP. Future studies should determine the optimal number of home BP measurements and identify the optimal parameter of home BPV needed to obtain reliable and valid estimates of the day-to-day home BPV.

\section{Conclusions}

This study has demonstrated that 24 weeks of azilsartan treat- 
ment after switching from another ARB significantly reduced the day-to-day BPV in the morning. Azilsartan also decreased the arterial stiffness, suggesting the mechanism underlying the reduction in home BPV. However, our study was unable to show whether the reduction in the home BPV by azilsartan can lead directly to better outcomes. Further studies are needed to determine this.

\section{Acknowledgments}

I would like to gratefully and sincerely thank Dr. Naoki Kondo for recruiting participants for this study.

\section{Funding Source}

This work was supported by JSPS KAKENHI Grant Number $15 \mathrm{~K} 09157$.

\section{Conflicts of Interest}

TM and HI received honoraria from Takada Pharmaceutical Co. The other authors have no conflicts of interest in relation to the materials presented in this article.

\section{References}

1. Stokes J, 3rd, Kannel WB, Wolf PA, D'Agostino RB, Cupples LA. Blood pressure as a risk factor for cardiovascular disease. The Framingham Study - 30 years of follow-up. Hypertension. 1989;13(5 Suppl):I13-18.

2. James PA, Oparil S, Carter BL, Cushman WC, Dennison-Himmelfarb C, Handler J, Lackland DT, et al. 2014 evidence-based guideline for the management of high blood pressure in adults: report from the panel members appointed to the Eighth Joint National Committee (JNC 8). JAMA. 2014;311(5):507-520.

3. Rothwell PM, Howard SC, Dolan E, O'Brien E, Dobson JE, Dahlof B, Sever PS, et al. Prognostic significance of visit-to-visit variability, maximum systolic blood pressure, and episodic hypertension. Lancet. 2010;375(9718):895905.

4. Parati G, Ochoa JE, Salvi P, Lombardi C, Bilo G. Prognostic value of blood pressure variability and average blood pressure levels in patients with hypertension and diabetes. Diabetes Care. 2013;36(Suppl 2):S312-324.

5. Verdecchia P, Angeli F, Gattobigio R, Rapicetta C, Reboldi G. Impact of blood pressure variability on cardiac and cerebrovascular complications in hypertension. Am J Hypertens. 2007;20(2):154-161.

6. Kikuya M, Ohkubo T, Metoki H, Asayama K, Hara A, Obara T, Inoue R, et al. Day-by-day variability of blood pressure and heart rate at home as a novel predictor of prognosis: the Ohasama study. Hypertension. 2008;52(6):1045-1050.
7. Muntner P, Whittle J, Lynch AI, Colantonio LD, Simpson LM, Einhorn PT, Levitan EB, et al. Visit-to-Visit Variability of Blood Pressure and Coronary Heart Disease, Stroke, Heart Failure, and Mortality: A Cohort Study. Ann Intern Med. 2015;163(5):329-338.

8. Webb AJ, Fischer U, Mehta Z, Rothwell PM. Effects of antihypertensive-drug class on interindividual variation in blood pressure and risk of stroke: a systematic review and meta-analysis. Lancet. 2010;375(9718):906-915.

9. Webb AJ, Wilson M, Lovett N, Paul N, Fischer U, Rothwell PM. Response of day-to-day home blood pressure variability by antihypertensive drug class after transient ischemic attack or nondisabling stroke. Stroke. 2014;45(10):2967-2973.

10. Turnbull F, Neal B, Pfeffer M, Kostis J, Algert C, Woodward M, Chalmers J, et al. Blood pressure-dependent and independent effects of agents that inhibit the renin-angiotensin system. J Hypertens. 2007;25(5):951-958.

11. Rakugi H, Enya K, Sugiura K, Ikeda Y. Comparison of the efficacy and safety of azilsartan with that of candesartan cilexetil in Japanese patients with grade I-II essential hypertension: a randomized, double-blind clinical study. Hypertens Res. 2012;35(5):552-558.

12. White WB, Weber MA, Sica D, Bakris GL, Perez A, Cao C, Kupfer S. Effects of the angiotensin receptor blocker azilsartan medoxomil versus olmesartan and valsartan on ambulatory and clinic blood pressure in patients with stages 1 and 2 hypertension. Hypertension. 2011;57(3):413420.

13. Ohshima K, Mogi M, Nakaoka H, Iwanami J, Min LJ, Kanno H, Tsukuda K, et al. Possible role of angiotensinconverting enzyme 2 and activation of angiotensin II type 2 receptor by angiotensin-(1-7) in improvement of vascular remodeling by angiotensin II type 1 receptor blockade. Hypertension. 2014;63(3):e53-59.

14. Kusumoto K, Igata H, Ojima M, Tsuboi A, Imanishi M, Yamaguchi F, Sakamoto H, et al. Antihypertensive, insulin-sensitising and renoprotective effects of a novel, potent and long-acting angiotensin II type 1 receptor blocker, azilsartan medoxomil, in rat and dog models. Eur J Pharmacol. 2011;669(1-3):84-93.

15. Iwai $M$, Chen $R$, Imura $Y$, Horiuchi M. TAK-536, a new AT1 receptor blocker, improves glucose intolerance and adipocyte differentiation. Am J Hypertens. 2007;20(5):579-586.

16. Matsumoto S, Shimabukuro M, Fukuda D, Soeki T, Yamakawa K, Masuzaki H, Sata M. Azilsartan, an angiotensin II type 1 receptor blocker, restores endothelial function by reducing vascular inflammation and by increasing the phosphorylation ratio $\operatorname{Ser}(1177) / \operatorname{Thr}(497)$ of endothelial nitric oxide synthase in diabetic mice. Cardiovasc Diabetol. $2014 ; 13: 30$.

17. Ogihara T, Kikuchi K, Matsuoka H, Fujita T, Higaki J, Horiuchi M, Imai Y, et al. The Japanese Society of Hypertension Guidelines for the Management of Hypertension (JSH 2009). Hypertens Res. 2009;32(1):3-107.

18. Kato T, Kikuya M, Ohkubo T, Satoh M, Hara A, Obara T, Metoki H, et al. Factors associated with day-by-day variability of self-measured blood pressure at home: the 
Ohasama study. Am J Hypertens. 2010;23(9):980-986.

19. Johansson JK, Niiranen TJ, Puukka PJ, Jula AM. Factors affecting the variability of home-measured blood pressure and heart rate: the Finn-home study. J Hypertens. 2010;28(9):1836-1845.

20. Matsui Y, O'Rourke MF, Hoshide S, Ishikawa J, Shimada K, Kario K. Combined effect of angiotensin II receptor blocker and either a calcium channel blocker or diuretic on day-by-day variability of home blood pressure: the Japan Combined Treatment With Olmesartan and a Calcium-Channel Blocker Versus Olmesartan and Diuretics Randomized Efficacy Study. Hypertension. 2012;59(6):1132-1138.

21. Imai Y, Otsuka K, Kawano Y, Shimada K, Hayashi H, Tochikubo O, Miyakawa M, et al. Japanese society of hypertension (JSH) guidelines for self-monitoring of blood pressure at home. Hypertens Res. 2003;26(10):771-782.

22. Miyoshi T, Ito H. Assessment of Arterial Stiffness Using the Cardio-Ankle Vascular Index. Pulse (Basel). 2016;4(1):11-23.

23. Ishikura K, Obara T, Kato T, Kikuya M, Shibamiya T, Shinki T, Ikeda U, et al. Associations between day-by-day variability in blood pressure measured at home and antihypertensive drugs: the J-HOME-Morning study. Clin Exp Hypertens. 2012;34(4):297-304.

24. Fukui M, Ushigome E, Tanaka M, Hamaguchi M, Tanaka T, Atsuta H, Ohnishi M, et al. Home blood pressure variability on one occasion is a novel factor associated with arterial stiffness in patients with type 2 diabetes. Hypertens Res. 2013;36(3):219-225.

25. Asayama K, Ohkubo T, Hanazawa T, Watabe D, Hosaka M, Satoh M, Yasui D, et al. Does Antihypertensive Drug Class Affect Day-to-Day Variability of Self-Measured Home Blood Pressure? The HOMED-BP Study. J Am Heart Assoc. 2016;5(3):e002995.

26. Ishiguro K, Hayashi K, Sasamura H, Sakamaki Y, Itoh H. "Pulse" treatment with high-dose angiotensin blocker reverses renal arteriolar hypertrophy and regresses hypertension. Hypertension. 2009;53(1):83-89.

27. Johansson JK, Niiranen TJ, Puukka PJ, Jula AM. Prognostic value of the variability in home-measured blood pressure and heart rate: the Finn-Home Study. Hypertension. 2012;59(2):212-218.

28. Matsui Y, Ishikawa J, Eguchi K, Shibasaki S, Shimada K, Kario K. Maximum value of home blood pressure: a novel indicator of target organ damage in hypertension. Hypertension. 2011;57(6):1087-1093.

29. Karpettas N, Destounis A, Kollias A, Nasothimiou E, Moyssakis I, Stergiou GS. Prediction of treatment-induced changes in target-organ damage using changes in clinic, home and ambulatory blood pressure. Hypertens Res. 2014;37(6):543-547.

30. Mena L, Pintos S, Queipo NV, Aizpurua JA, Maestre $G$, Sulbaran T. A reliable index for the prognostic significance of blood pressure variability. J Hypertens. 2005;23(3):505-511. 